\title{
What makes pediatric clinical research ethical?
}

If we asked an audience made up of researchers working with human beings or members from research ethics committees what comes to their minds when speaking about research ethics, "informed consent" would be the most common answer.

Such answer is undoubtedly related to the historical focus placed on autonomy as an indispensable requirement for ethical research.

It appears that such supremacy of autonomy may be partially responsible for the difficulties encountered by research on children and adolescents.

Specifically leaving aside of this discussion research on minors, it could be said that consent is not always a necessary or sufficient condition to ensure that research is conducted ethically. Sometimes, with retrospective studies with unidentified data, anonymous surveys, or studies performed in situations of an urgency or unconsciousness of patients, a consent is unnecessary or unfeasible; and, as long as subjects' rights are respected, a study can be conducted in an ethically successful manner.

So, what makes human clinical research ethical? Ezekiel Emanuel provides a very clear explanation. For research to be ethical, in the first place it requires social value. Research work that is not aimed at producing valuable knowledge for society is ethically unacceptable. Examples of research without social value are redundant, these are studies conducted to look into "me too" drugs. Research with no social value only squanders resources and exposes participants to unnecessary risks and discomforts.

Secondly, research requires scientific validity. The proposed study design, materials and methods should reasonably ensure that the question posed in the study objective will be answered. If not warranted, again, it means squandering resources, exposing participants, using the consent to "promise" participants that, thanks to their involvement, science will find new answers,etc. When a study has no scientific validity, research ethics is undermined even before the consent is brought into the picture. For this reason, committees established to assess research should be trained to evaluate methodological aspects related to design, sampling, sample size and data analysis.
A third requirement is related to the fair selection of the study population. This implies that groups selected for study should be related to the research question. Many times, participants are selected "by convenience" and not because research is focused on finding a solution to the problem of the group.

It is quite likely that this situation of exploitation of vulnerable groups, which many times include children, is also a reason for the self-imposed censorship on research conducted on minors.

In addition, a fair selection of subjects entails that all groups are offered the opportunity to participate in research unless there are good scientific or risk-related reasons restricting such eligibility. In other words, vulnerable groups or those with restricted autonomy should not be used due to "convenience" and because they are susceptible to coercion; but at the same time, it is necessary not to restrict the right of subjects to participate in research just because they are part of a vulnerable population. Screening can only be fair when those enrolled as subjects are likely to benefit from research.If a drug or procedure is likely to be used on children and proves to be safe and effective, then fairness requires including them. Actually, one of the fundamental reasons for the recent initiatives of including more women, minorities and children in clinical research is to ensure that they are enrolled as research subjects in interventions that might be used on them in the future.

This critical aspect of ethical research, a fair selection of the sample, is the foundation for including children in studies aimed at answering valuable and scientifically valid questions for this group.

From an ethical perspective, when it is proposed to enroll children in a study, the relevant question to be answered should be: "Are children and adolescents included for scientific reasons, because the study question is related to this age group? Or are children and adolescents being enrolled because they are easily manipulated and 'convenient'?" If the answer corresponds to the first option, then their inclusion is absolutely necessary.

If they are not included, we might be unprotecting those we have chosen to protect and 
leaving children and adolescents in a therapeutic neglect which they have been historically subjected to.

Another requirement for research to be ethical is an acceptable risk-benefit ratio maximizing participants' benefits and minimizing their risks.

The next requirement is an independent evaluation. All research results in a potential conflict of interest, either financial or academic. A conflict of interest occurs when the primary interest, in this case, the aim of a doctor in achieving the cure or relief of patients, collides with a secondary interest such as the financial or academic benefit of conducting a study. This conflict of interest supports the essential requirement of having an independent research ethics committee review every project whether being observational or posing a minimum risk.

Only when all those requirements are met, the informed consent comes into the picture. If conditions listed here are not warranted, the informed consent is of no use. In other words, no research can be considered ethical if it lacks social value, has an invalid methodology, the sample selection is unfair, and benefits are disproportionate to risks, no matter how spectacular the informed consent procedure is.

In the case of minors, from a strictly legal perspective, it is parents who give their consent. Children should be informed and listened to as to whether they want to participate. This procedure is called assent and should be adapted based on the child's maturity.

Lastly, for research to be ethical, it should respect participants ensuring their well-being; this means, informing them of any new studyrelated finding and compensating them in case of suffering any damage as a result of the study.

A requirement which usually does not receive much attention because it takes place once the study has ended is data publication. Social value in research and its essence are attacked if what has been studied is left unpublished. Not publishing the data collected is a fraud to participants who have been promised that thanks to their participation scientific knowledge will expand. This can only occur if the information obtained is disseminated.

The Royal College of Pediatrics and Child Health has recently updated its Guidelines for the ethical conduct of medical research involving children. The Guidelines consider that research involving children is important and that principles therein listed should be met. They underscore that children have rights, including the right to participate in a research study, with adequate protection, minimizing risks and optimizing benefits.

The Guidelines state that research that involves greater than minimal risk may be acceptable provided benefits, if any, are allocated to them. They also point out that drugs should be previously tested on adults. There is a section in the Guidelines that deals with the assent procedure and it highlights that a child's legal status may change when projects go on for several years.

In relation to research conducted in children under situations of urgency, the Guidelines clearly state that research is absolutely necessary and that it should be conducted if the selection of this population is strictly linked to the research question and such question cannot be otherwise answered.

Likewise, the Guidelines remind us that research on children should be conducted by staff qualified in managing the problem being studied, and also by a team trained in Good Clinical Practice in Research. Both are essential requirements that cannot be overlooked.

In short, research on children is necessary. In order to conduct it, universal requirements that ensure ethical research should be met.

Karin Kopitowski, M.D.

Head of Family and Community Medicine Service Coordinator of the Research Protocol Ethics Committee (CEPI) Hospital Italiano de Buenos Aires

http:/ /dx.doi.org/10.5546/aap.2014.eng.492

\section{REFERENCES}

1. Emanuel EJ, Wendler D, Grady C. What makes clinical research ethical? JAMA 2000;283(20):2701-11.

2. Levine C, Faden R, Grady C, Hammerschmidt D, et al. The limitations of "vulnerability" as a protection for human research participants. Am J Bioeth 2004;4(3):44-9.

3. ModiN, Vohra J,Preston J,ElliottC, etal.Guidance on clinical research involving infants, children and young people: an update for researchers and research ethics committees. Arch Dis Child 2014 Jun 9. pii: archdischild-2014-306444. [Epub ahead of print]. 